\title{
Mejora de la capacidad de salto en mujeres postmenopáusicas con moderado riesgo de fractura de cadera tras dos años de ejercicio en el medio acuático Results of two years of water training on jump height in postmenopausal women with moderate hip risk fracture
}

\author{
*María Carrasco Poyatos, Manuel Vaquero Abellán \\ *Universidad de Murcia (España), **Universidad de Córdoba (España)
}

\begin{abstract}
Resumen: La finalidad del presente estudio fue establecer el efecto de un programa de impacto y resistencias adicionales en el medio acuático sobre la capacidad de salto en mujeres postmenopáusicas con moderado riesgo de fractura de cadera. 39 mujeres fueron asignadas a tres grupos: grupo de natación (GN; $n=17)$, grupo de impacto y resistencias (GIR; $n=14)$ y grupo control $(\mathrm{GC} ; \mathrm{n}=8$ ). Se llevó a cabo un test de composición corporal, mediante el que se analizó el índice de masa corporal (IMC) y el índice cintura-cadera (ICC); y un test de capacidad de salto, en el que se realizó un salto con contramovimiento (CMJ). GN mostró un descenso significativo ( $\mathrm{p}<0.05)$ en el ICC (5.81\%). GIR mejoró significativamente el IMC (3.65\%) y el CMJ $(15.5 \%)$. Dos años de ejercicio de impacto y resistencias adicionales en el medio acuático pueden mejorar la capacidad de salto de mujeres postmenopáusicas con moderado riesgo de fractura de cadera. Tanto este programa de ejercicio como el de natación pueden ofrecer cambios positivos en la composición corporal de dichas mujeres.
\end{abstract}

Palabra clave: postmenopausia, capacidad de salto, medio acuático, composición corporal.

Abstract: The aim of the present study was to investigate the effect of a water-based calisthenics and resistance program on jump height in postmenopausal women with moderate hip risk fracture. 39 women were divided into three groups: swimming group (GN; $n=17)$, calisthenics and resistance group (GIR; $\mathrm{n}=14)$, and control group $(\mathrm{GC} ; \mathrm{n}=8)$. Body composition test included body mass index (IMC) and waist to hip ratio (ICC). Jump height was assessed by a countermovement jump (CMJ). GN showed a significant $(\mathrm{p}<0.05)$ decrease in ICC $(5.81 \%)$. GIR showed a significant decrease in IMC (3.65\%) and a significant increase in CMJ (15.5\%). Two years of water-based calisthenics and resistance training can offer significant benefits in jump height in postmenopausal women with moderate hip risk fracture. Both exercise programs can also improve body composition. Key words: postmenopausal period, jump height, resistance training, body compositions.

\section{Introducción}

La menopausia es un proceso fisiológico degenerativo celular transcrito en el código genético de la mujer caracterizado por la reducción de la producción hormonal (estrógenos y progesterona), la desaparición de la ovulación y la finalización el ciclo menstrual (Greeves, Cable, Reilly, \& Kingsland, 1999; Muntané, 1994). Afecta al 90\% de las mujeres y suele manifestarse alrededor de los 51.4 años, con un espectro que va de los 45 a los 55 años (Asikainen, Kukkonen-Harjula, \& Miilunpalo, 2004; Bonita, 1998; Laskin, Costlow, López, Taub, \& Kronenberg, 1994; Muntané, 1994).

La reducción de la producción hormonal origina cambios a nivel estructural y funcional en el organismo de la mujer. Es frecuente que las mujeres postmenopáusicas padezcan osteoporosis, un estado patológico de fragilidad ósea aumentada causado por la reducción vertiginosa de la densidad de masa ósea y el deterioro de la microarquitectura del hueso (Cenci et al., 2000; Humphries et al., 1999; Riggs, Khosla, \& Melton, 1998). A su vez, ésta está íntimamente relacionada con el riesgo de fractura de cadera (Bouxsein, Coan, \& Lee, 1999; Hans, Hartl, \& Krieg, 2003; Stewart, Kumar, \& Reid, 2006), que es la mayor causa de pérdida funcional, dependencia y riesgo de muerte en los mayores (Keene, Parker, \& Pryor, 1993; Meunier et al., 1999). Por otro lado, el sistema neuromuscular también se ve afectado por estos cambios hormonales. Diversos estudios han encontrado disminuciones significativas en la fuerza y la masa muscular tras la menopausia (Humphries et al., 1999; Kamel, Maas, \& Duthie, 2002; Van Langendonck, Claessens, Lysens, Koninckx, \& Beunen, 2004). Con la edad, se produce un deterioro de la conducción nerviosa motora del sistema nervioso periférico (Connelly, Rice, Roos, \& Vandervoort, 1999; Daley \& Spinks, 2000) y de la capacidad de reclutamiento de unidades motoras (Klass, Baudry, \& Duchateau, 2008), en concreto son las fibras musculares rápidas (Tipo

Fecha recepción: 25-11-09 - Fecha envío revisores: 25-11-09 - Fecha de aceptación: 20-12-09 Correspondencia: María Carrasco Poyatos

Facultad de Ciencias del Deporte. Universidad de Murcia.

C/ Argentina, s/n. 30720 Santiago de la Rivera- San Javier. Murcia. España.

Email: mariacarrasco@um.es
II oFT) las que se ven más afectadas por este fenómeno tendiendo a ser reabsorbidas por el organismo, lo que origina una proporción más elevada de fibras lentas (Wilmore \& Costill, 2004) y por tanto la ralentización de los movimientos. Según algunas investigaciones (Kent-Braun \& Ng, 1999), no está claro que en las mujeres el nivel de estrógenos afecte a la contracción muscular voluntaria, aunque sí se observa su relación con la ralentización en la velocidad de movimiento segmentaria.

Los cambios del sistema neuromuscular tienen un impacto particularmente severo en la autonomía e independencia de las mujeres postmenopáusicas, siendo en parte responsables del aumento de las caídas (Runge \& Schacht, 2005; WHO \& TUSNP, 2002) y disminuyendo la habilidad para realizar las actividades instrumentales de la vida cotidiana (Kell, Bell, \& Quinney, 2001), como bajar o subir escaleras o levantarse y sentarse en el sofá. Por este motivo pueden llegar a ser una peligrosa amenaza para la capacidad funcional, la salud y la calidad de vida.

Dada la fragilidad ósea de la muestra, en el presente estudio se eligió el medio acuático para llevar a cabo los programas de ejercicio ya que, debido a sus características de flotación y resistencia, por un lado aporta confianza y seguridad en la ejecución de movimientos eliminado en mayor medida el incipiente riesgo de caídas y fracturas que caracteriza a este tipo de población, y por otro, este medio ofrece la posibilidad de trabajar la fuerza y la resistencia muscular con y sin implementos adicionales, manteniendo el control de la intensidad del ejercicio físico realizado.

Investigaciones previas indican que el trabajo de impacto y resistencias adicionales en piscina poco profunda parece repercutir de manera positiva en la capacidad de salto de mujeres postmenopáusicas y en algunos componentes relacionados con la composición corporal (Takeshima et al., 2002; Tsourlou, Benik, Zafeiridis, \& Kellis, 2006). Otros estudios basados en ejercicios pliométricos tanto en el medio acuático (Martel, Harmer, Logan, \& Parker, 2005) como aéreo (Sipilä et al., 2001; Taaffe et al., 2005), también obtienen resultados positivos en la capacidad de salto. Por consiguiente, el objetivo del presente estudio fue analizar el efecto de un programa de impacto y resistencias adicionales en el medio acuático sobre la capacidad de salto en mujeres 
postmenopáusicas con moderado riesgo de fractura de cadera, estableciendo las diferencias con respecto a un programa basado en actividades propias de la natación y a un grupo control.

\section{Material y método}

\subsection{Descripción de la muestra}

En el presente estudio participaron 93 mujeres $(n=93)$ postmenopáusicas con moderado riesgo de fractura de cadera. Fueron reclutadas de las consultas externas de atención primaria y especializada del Centro de Salud de Palomarejos y del Centro Especializado de Diagnóstico y Tratamiento del SESCAM de Toledo (España). Los criterios de inclusión que debían cumplir las participantes fueron los siguientes: mujeres postmenopáusicas de Toledo capital con al menos 5 años de amenorrea, edad menor o igual a 70 años, con moderado riesgo de fractura de cadera medida por ultrasonografía de calcáneo con el densitómetro Aquiles Express 2000 (General Electric, Lunar Corporation) y siguiendo las recomendaciones de Hans et al: $-1,2<$ QUS T-score > -2,5 (Hans et al., 2003), sin antecedentes de fractura osteoporótica, que no utilizaran la Terapia Hormonal Sustitutiva como tratamiento para la osteoporosis, sin patologías neurológicas, musculoesqueléticas, cardiovasculares, metabólicas o inflamatorias que les impidieran su participación en los programas de ejercicio en el medio acuático. Ninguna de ellas estuvo incluida en algún programa de ejercicio en el medio acuático en los dos años anteriores al presente estudio. Las participantes que no cumplieron dichos criterios fueron excluidas.

La muestra fue dividida en 3 grupos: grupo de natación (GN), grupo de impacto y resistencias (GIR) y grupo control (GC). GN estaba formado por 17 mujeres, GIR por 14, y GC por 8. Cada participante firmó un formulario de consentimiento tras ser informadas de los riesgos y beneficios de su participación en el estudio. Asimismo, todas recibieron instrucciones de no alterar sus hábitos alimentarios y de mantener la práctica de actividad física habitual durante el periodo de entrenamiento. De las 93 mujeres iniciales, 39 completaron el estudio y fueron incluidas en el análisis final. Las bajas de produjeron por motivos familiares y otro tipo de patologías no relacionadas con el estudio. Las características de las participantes se muestran en la tabla 1.

\subsection{Test y protocolos de aplicación}

Todas las mediciones fueron llevadas a cabo en el Laboratorio de Entrenamiento Deportivo de la Facultad de Ciencias del Deporte de Toledo, Universidad de Castilla-La Mancha(España). Para ello se empleó siempre el mismo equipamiento, los mismos evaluadores y las mismas técnicas de evaluación. Los evaluadores fueron adiestrados y estaban perfectamente cualificados para desempeñar su labor. Las mediciones antropométricas fueron realizadas siempre antes de la medición de la capacidad de salto y ambas se llevaron a cabo siempre el mismo día. El test de capacidad de salto se hizo en la semana previa a la del pretest y el postest para minimizar el efecto del aprendizaje. El mejor valor de cada test fue utilizado en el análisis estadístico.

Determinación de la composición corporal. Las mediciones de peso y talla fueron realizadas por el mismo evaluador a todas las participantes para posteriormente hallar el índice de masa corporal (IMC). Para ello se utilizó una báscula médica electrónica SECA 780 con tallímetro. Para medir el índice cintura-cadera (ICC) se utilizó una cinta métrica Harpender Anthropometric (Holtain LTD). Las participantes fueron medidas descalzas y con ropa ligera.

Medición de la capacidad de salto. Se eligió el test de salto con contramovimiento o contermovement jump (CMJ) por ser un salto sencillo de ejecutar en el que, mediante la flexo-extensión de piernas, se aprovecha su carácter elástico-explosivo para una mayor elevación del centro de gravedad, dado que el trabajo excéntrico (flexión) que precede al concéntrico (extensión) hace que se almacene energía elástica que será reutilizada en la fase de empuje. Así mismo, la fase excéntrica permite que los sujetos con un porcentaje alto de fibras lentas tengan más

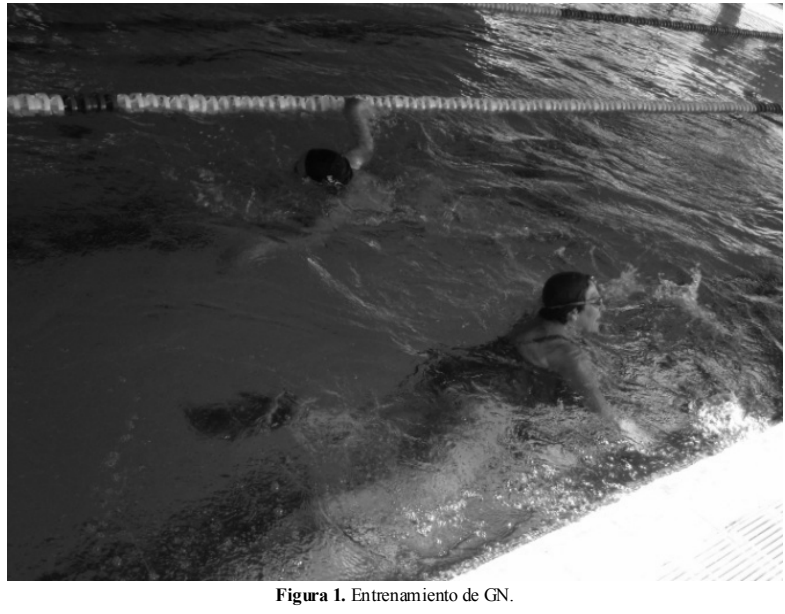

tiempo para reclutar fibras rápidas (Bosco, 1994), lo que se adecúa a las características de la muestra del presente estudio. La ejecución de este salto consiste en, desde posición erecta, llegar hasta una semiflexión de rodilla aproximada de $90^{\circ}$-intentando que el tronco esté lo más recto posible para evitar su influencia en el movimiento de las piernas (Bosco, 1994)-y realizar una extensión de piernas intentando llegar lo más alto posible sin la ayuda de los brazos. El periodo de tiempo entre la fase excéntrica y la concéntrica debe ser corto para que la energía elástica acumulada se aproveche en el salto. Para llevar a cabo el CMJ se utilizó el Ergo-Jump Bosco System. Este instrumento parte del tiempo de vuelo para el cálculo directo de la elevación del centro de gravedad (Bosco, 2000), midiendo la altura del salto en centímetros. Se compone de una plataforma de infrarrojos conectada a un sistema de cronometraje electrónico que es accionado automáticamente por el mismo sujeto que salta en el momento del despegue, cerrándolo en el momento en el que el pie contacta otra vez con el terreno. Lleva siendo utilizado por federaciones, clubes, centros de investigacióny Facultades Universitarias desde hace mucho tiempo para diferentes trabajos técnicos y científicos. Previamente a la realización del test se llevó a cabo un calentamiento compuesto por 5 minutos en cicloergómetro (Monark Ergomedic 828E) a 25 wats y 5-7 ensayos del CMJ fuera de la plataforma con el objetivo de asegurar una ejecución correcta del mismo. Para la realización del test de CMJ, las participantes se situaron en la plataforma de infrarrojos, entre el emisory el receptor, con pies a la anchura de las caderas y manos en la cintura. Tras la indicación del evaluador («preparadas, ¡ya!») realizaron el CMJ. El evaluador controló que la ejecución técnica del salto fuera correcta en la realización del test. El test se repitió dos veces, obteniéndose el valor máximo de los dos intentos. Este procedimiento ha sido validado por su utilización en varios estudios (Cottini et al., 1996; Vilijanen, Viitasalo, \& Kujala, 1991).

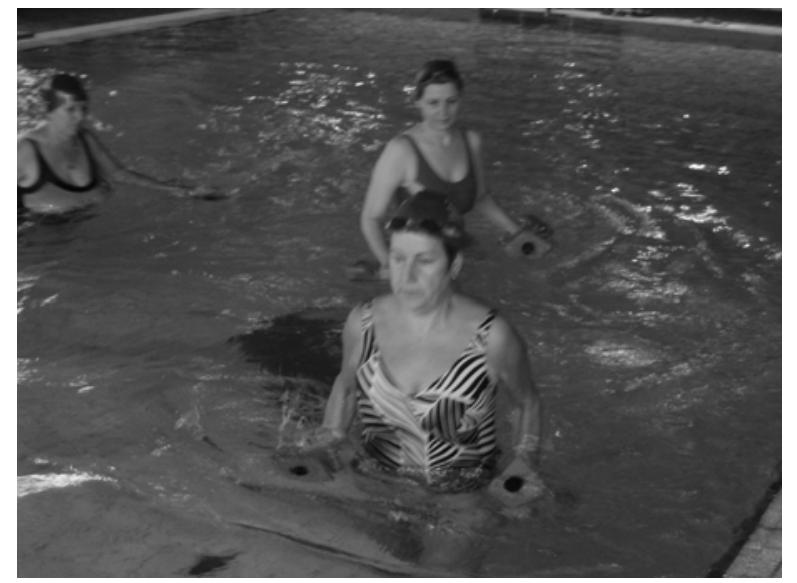

Figura 2. Entrenamiento de GIR 


\subsection{Intervención}

Los grupos de ejercicio (GNy GIR) llevaron a cabo un programa de entrenamiento durante 24 meses con una frecuencia de 2 días/semana y una duración de 45 minutos/sesión. Todas las sesiones de ejercicio fueron desempeñadas y supervisadas por un entrenador cualificado. Todas las participantes cumplieron un mínimo de asistencia del 95\%. Ninguna mujer sufrió algún tipo de lesión en las sesiones de entrenamiento.

Siguiendo las recomendaciones del American College of Sports Medicine (ACSM) (Pollock et al., 1998), los programas de ejercicio se realizaron a una intensidad moderada. Para determinarla, y siguiendo a otros estudios con mujeres postmenopáusicas en el medio acuático (Ay \& Yurtkuran, 2005), se utilizó la Escala de Esfuerzo Percibido de Borg. Para permitir la adaptación progresiva de las participantes al entrenamiento, la cantidad de trabajo fue incrementándose de manera progresiva cada 3 meses aproximadamente. Durante el primer año, las participantes entrenaron dentro de un rango de intensidad ligera-moderada según la puntuación de esfuerzo percibido 10-13 en la Escala de Borg. Durante el segundo año, entrenaron dentro de un rango de intensidad moderada según la puntuación de esfuerzo percibido 13-15 en la Escala de Borg.

Las sesiones de ejercicio de GN consistieron en ejercicios propios de la natación en piscina profunda, sin apoyo de los pies en el suelo y predominantemente en posición horizontal del cuerpo, en los que se utilizaron implementos típicos de la natación como aletas o palas para incrementar la intensidad de trabajo (figura 1). Todas las sesiones incluyeron un periodo de calentamiento ( 5 minutos) que consistía en nado libre a baja intensidad, el programa principal (35 minutos), y un periodo de vuelta a la calma ( 5 minutos) en el que se realizaron estiramientos globales en piscina poco profunda.

Las sesiones de ejercicio de GIR consistieron en ejercicios de impacto del tren inferior y resistencias adicionales del tren superior e inferior en piscina poco profunda, como acuaeróbic, step, circuitos de musculación o juegos, realizados predominantemente en posición vertical del cuerpo, con el nivel del agua por la cintura y apoyo de los pies en el suelo (figura 2). Para aumentar la intensidad de trabajo en el tren inferior y superior se utilizaron materiales específicos (Leisis, S.L. Valencia. Spain). Para el tren inferior: water weight boots. Para el tren superior: minifins, hydrobells $\mathrm{y}$ water weight bells. Todas las sesiones incluyeron un periodo de calentamiento (5 minutos) que consistía en actividades lúdicas de baja intensidad, el programa principal (35 minutos), y un periodo de vuelta a la calma ( 5 minutos) en el que se realizaron estiramientos globales en piscina poco profunda.

\subsection{Análisis estadístico de los datos}

Tras asegurar una distribución normal de las variables se realizó un análisis descriptivo. Para comparar grupos en bloque (conjunto) se utilizó el análisis de la varianza (ANOVA). El test de Bonferroni fue aplicado para determinar diferencias específicas entre medias. Para analizar los cambios de cada variable en cada grupo se utilizó la prueba de la t para muestras relacionadas. Se aplicó un margen de error de $\mathrm{p}<$ 0.05 . Los datos fueron analizados con paquete estadístico SPSS (version 15.0, SPSS Inc, Chicago, IL).

\section{Resultados}

Como se muestra en la tabla 2 , al final del estudio las participantes de GN redujeron de manera significativa el ICC $(-0.05 \mathrm{~cm})$, y las participantes de GIR, el IMC $\left(-0.97 \mathrm{~kg} / \mathrm{m}^{2}\right)$. No se encontraron diferencias estadísticas entre grupos en las variables antropométricas. En cuanto a la capacidad de salto, se obtuvieron mejoras significativas en el CMJ de GIR $(2.52 \mathrm{~cm})$, aunque no se encontraron diferencias estadísticas entre grupos en relación a la capacidad de salto.

\section{Discusión}

Los hallazgos más notables de esta investigación muestran que dos años de trabajo de impacto y resistencias adicionales en el medio acuático son efectivos a la hora de producir mejoras significativas en la capacidad de salto de mujeres postmenopáusicas con moderado riesgo de fractura de cadera. Aunque no existan diferencias significativas con respecto a GN y GC, es evidente que las características del ejercicio realizado por GIR son adecuadas para producir dichas mejoras. Por otro lado, si el volumen de ejercicio se incrementara siguiendo las recomendaciones del ACSM(3 días/semana, 60 minutos/sesión) (Ashe \& Khan, 2004; Pollock et al., 1998), posiblemente se obtendrían diferencias significativas entre grupos.

En relación a estos resultados, el presente estudio coincide con otros de similares características realizados con mujeres postmenopáusicas durante 3 y 6 meses respectivamente (Takeshima et al., 2002; Tsourlou et al., 2006) y en los que se ha valorado la capacidad de salto mediante la realización de un CMJ y utilizando el mismo instrumento que en el presente estudio (Ergo Jump Bosco System).

ElCMJ está relacionado con el componente explosivo de la fuerza que ejerce la musculatura flexo-extensora de las piernas (fuerza elásticoexplosiva)(Vanezis \& Lees, 2005), utilizando las articulaciones de tobillos, rodillas y cadera como componentes de la cadena cinética. Pero además de la fuerza muscular, en la realización del salto influyen otros factores a nivel neuromuscular que hacen más eficiente la ejecución del mismo, como el estado de entrenamiento en el que se encuentra la fibra muscular y el tipo de fibra reclutada (Bosco, 1994), la capacidad de imprimir mayor fuerza muscular en un movimiento rápido y la activación y coordinación intermuscular de los músculos sinergistas (Vanezis \& Lees, 2005), la inhibición de los músculos antagonistas, o la excitabilidad y el feedback tanto neuronal (Deschenes \& Kraemer, 2002) como de los componentes encargados en la realización del movimiento a nivel muscular (Bosco, 1994). Estos factores van a ser determinantes en la eficacia del salto y están íntimamente relacionados con la calidad en la ejecución del gesto técnico (Bobbert \& Van Soest, 1994) y con del tipo de entrenamiento llevado a cabo (Gehri, Ricard, Kleiner, \& Kirkendall, 1998; Markovic, 2007; Potteiger et al., 1999). El entrenamiento pliométrico al que se le añade un trabajo adicional de fuerza muscular es la forma más efectiva de mejorar la capacidad de salto y la fuerza del tren inferior (Chirosa, Chirosa, Requena, Feriche, \& Padial, 2002; Ebben, 2002).

Siendo conscientes de la dificultad que entraña mejorar el gesto técnico en cualquier disciplina y que para la optimización del gesto técnico del salto son necesarias varias semanas consecutivas de práctica (Bobbert \& Van Soest, 1994), no es probable que las participantes de GIR hayan obtenido incrementos en la capacidad de salto por haber mejorado su ejecución técnica. Sin embargo, el tipo de entrenamiento llevado a cabo por este grupo sí ha podido tener mucho que ver con los resultados dado que su entrenamiento estuvo basado en saltos en todas sus formas, con variaciones angulares y velocidades variables, saltos a la pata coja, subidas a un escalón con carga adicional, zancadas con carga adicional, siempre con apoyo de los pies en el suelo y manteniendo la posición de pie. Este tipo de ejercicios han sido indicados para la mejora

\begin{tabular}{|c|c|c|c|}
\hline \multicolumn{4}{|c|}{ Tabla 1. Características de la muestra (media \pm DT). } \\
\hline & $\begin{array}{c}\mathrm{GN} \\
(\mathrm{n}=17)\end{array}$ & $\begin{array}{c}\mathrm{GIR} \\
(\mathrm{n}=14)\end{array}$ & $\begin{array}{c}\mathrm{GC} \\
(\mathrm{n}=8)\end{array}$ \\
\hline Edad (años) & $59.4 \pm 6.2$ & $56.5 \pm 6.4$ & $57.3 \pm 6.5$ \\
\hline Altura $(\mathrm{cm})$ & $156.3 \pm 6.8$ & $155.5 \pm 6.4$ & $154.2 \pm 5.4$ \\
\hline Peso (kg) & $61.1 \pm 7.7$ & $64.4 \pm 15.7$ & $64.3 \pm 10.9$ \\
\hline $\operatorname{IMC}\left(\mathrm{kg} / \mathrm{m}^{2}\right)$ & $24.9 \pm 2.4$ & $26.5 \pm 5.6$ & $27.2 \pm 5.5$ \\
\hline $\operatorname{ICC}(\mathrm{cm})$ & $0.81 \pm 0.0$ & $0.82 \pm 0.1$ & $0.8 \pm 0.1$ \\
\hline \multicolumn{4}{|c|}{ Tabla 2. Medidas antropométricas y CMJ en los tres grupos (media \pm DT). } \\
\hline \multicolumn{4}{|l|}{$\mathrm{IMC}\left(\mathrm{kg} / \mathrm{m}^{2}\right)$} \\
\hline Pre & $25.92 \pm 2.5$ & $26.55 \pm 3.5$ & $27.58 \pm 6.2$ \\
\hline Post & $26.07 \pm 2.3$ & $25.58 \pm 2.9 *$ & $25.03 \pm 2.4$ \\
\hline \multicolumn{4}{|l|}{$\operatorname{ICC}(\mathrm{cm})$} \\
\hline Pre & $0.86 \pm 0.0$ & $0.84 \pm 0.1$ & $1.54 \pm 2.0$ \\
\hline Post & $0.81 \pm 0.0^{*}$ & $0.76 \pm 0.2$ & $0.79 \pm 0.0$ \\
\hline \multicolumn{4}{|l|}{$\mathrm{CMJ}(\mathrm{cm})$} \\
\hline Pre & $13.76 \pm 3.3$ & $13.74 \pm 2.8$ & $12.10 \pm 3.4$ \\
\hline Post & $21.22 \pm 20.3$ & $16.26 \pm 4.4^{*}$ & $12.64 \pm 5.2$ \\
\hline \multicolumn{4}{|c|}{$\begin{array}{c}\text { *Diferencia significativa respecto a la valoración inicial }(\mathrm{p}<0.05) \\
\text { IMC }=\text { indice de masa corporal. } \mathrm{ICC}=\text { indice cintura-cadera. } \\
\mathrm{CMJ}=\text { salto con contramovimiento. } \mathrm{GN}=\text { grupo de natación. } \\
\mathrm{GIR}=\text { grupo de impacto y resistencias. } \mathrm{GC}=\text { grupo ontrol. }\end{array}$} \\
\hline
\end{tabular}


de la ejecución del CMJ (Bosco, 1994), hecho que además puede incidir sobre los procesos neuromusculares y las propiedades viscoelásticas de los músculos a la hora de realizar el salto. Por el contrario, con el programa de ejercicio que realizó el GN no se han obtenido cambios significativos en la capacidad de salto, presumiblemente por la posición horizontal del cuerpo y la inexistencia de saltos en su programa de entrenamiento.

Debido a estas afirmaciones, en el presente estudio no se puede relacionar el incremento significativo de la capacidad de salto de GIR únicamente con la mejora de la fuerza explosiva del tren inferior, sino que en la obtención de estos resultados también habrá influido la mejora de los factores neuromusculares anteriormente descritos. Estos resultados son un hallazgo importante ya que se demuestra que con el ejercicio de impacto y resistencias adicionales en el medio acuático es posible mejorar la fuerza explosiva del tren inferior y los factores neuromusculares asociados a la ejecución del salto en mujeres que, tras pasar la menopausia, van a acusar más la incidencia del paso del tiempo sobre la capacidad estructural y funcional del sistema neuromuscular. Por tanto, este ejercicio ofrece la posibilidad de mejorar el estado funcional y por consiguiente, la salud y la calidad de vida de mujeres postmenopáusicas con moderado riesgo de fractura de cadera.

Por otro lado, en ambos grupos de ejercicio (GN y GIR) se han obtenido mejoras significativas en alguno de los componentes relacionados con la composición corporal. En GN se encontraron mejoras significativas en el ICC, y en GIR, en el IMC. Aunque no existen diferencias significativas con respecto a GC en ninguno de los dos grupos. Estos cambios son similares a los obtenidos por otros estudios tras 4-6 meses de entrenamiento de natación o de impacto y resistencias adicionales en el medio acuático con hombres y mujeres de mediana edad (Colado, Triplett, Tella, Saucedo, \& Abellán, 2009; Cox et al., 2008; Cox etal., 2006; Volaklis, Spassis, Tokmakidis, \& Greece, 2007). En el estudio de Takeshima et al. (2002) anteriormente mencionado, también se encontraron mejoras significativas en la composición corporal, aunque el método de medición utilizado (suma de pliegues) no coincide con el del presente estudio. Por tanto, y sin la intención de hacer afirmaciones definitivas sobre los cambios en la composición corporal, tanto con la natación como con el trabajo de impacto y resistencias adicionales en el medio acuático se pueden obtener mejoras en la composición corporal de mujeres postmenopáusicas con moderado riesgo de fractura de cadera, otro factor más que contribuye en la mejora de la salud y la calidad de vida. Estos resultados son interesantes ya que normalmente los cambios antropométricos derivados del ejercicio suelen producirse en hombres, especialmente en personas jóvenes (Fiatarone, 2002), a lo que se le suma que tras la menopausia es más complicado mejorar la composición corporal (Van Pelt et al., 1998).

\section{Conclusiones}

El objetivo principal del presente estudio fue mejorar la capacidad de salto de mujeres postmenopáusicas con moderado riesgo de fractura de cadera para incidir de manera positiva en su capacidad de realizar las actividades instrumentales de la vida cotidiana y por tanto mejorar su salud y calidad de vida. Aunque los cambios obtenidos en la capacidad de salto en GIR no sean significativamente diferentes a los obtenidos en GN y GC y no se pueda afirmar cual de los tres grupos es mejor, es indudable que el programa de ejercicio de impacto y resistencias adicionales en el medio acuático de dos años de duración ha originado mejoras en la capacidad de salto de mujeres postmenopáusicas con moderado riesgo de fractura de cadera. Adicionalmente, tanto con el programa de impacto y resistencias adicionales como con el de natación se han obtenido cambios positivos en la composición corporal de dichas mujeres. En conclusión, utilizar el medio acuático para realizar un entrenamiento de impacto con personas que tienen riesgo de fractura ósea en el medio aéreo, ofrece beneficios significativos tanto en la capacidad de salto como en la composición corporal.

\section{Agradecimientos}

Los resultados del presente estudio pertenecen al Proyecto Osteoaqua: estudio de la eficacia de un programa de actividad física en el medio acuático en la prevención y tratamiento de la osteoporosis en mujeres postmenopáusicas, que se realizó en la Facultad de Ciencias del Deporte de Toledo, Universidad de Castilla-La Mancha (España). El director principal de dicho proyecto fue Dr. Fernando Navarro Valdivielso. La financiación del mismo corrió a cargo de la Junta de Comunidades de Castilla-La Mancha.

\section{Referencias bibliográficas}

Ashe, M. C., \& Khan, K. M. (2004). Exercise prescription. Journal of the American Academy of Orthopaedic Surgeons, 12, 21-27.

Asikainen, T. M., Kukkonen-Harjula, K., \& Miilunpalo, S. (2004). Exercise for health for early postmenopausal women. Sports Medicine, 34(11), 753-778.

Ay, A., \& Yurtkuran, M. (2005). Influence of aquatic and weightbearing exercises in quantitative ultrasound variables in postmenopausal women. American Journal of Physical Medicine \& Rehabilitation, 84(1), 10.

Bobbert, M. F., \& Van Soest,A. J.(1994). Effects on muscle strengthening on vertical jump height: a simulation study. Medicine and Science in Sports and Exercise, 26(8), 8.

Bonita, R. (1998). Mujeres, envejecimiento y salud. Conservar la salud a lo largo de la vida [Electronic Version]. Organización Mundial de la Salud. http://www.imsersomayores.csic.es/documentos/ documentos/bonita-mujeres-01.pdf, 67. Retrieved 12-08-08,

Bosco, C. (1994). La valoración de la fuerza con el test de Bosco (1 $1^{\mathrm{a}}$ ed.). Barcelona: Editorial Paidotribo.

Bosco, C. (2000). La fuerza muscular. Aspectos metodológicos. Barcelona: Editorial Inde.

Bouxsein, M. L., Coan, B. S., \& Lee, S. C. (1999). Prediction of the strength of the elderly proximal femur by bone mineral density and quantitative ultrasound measurements of the heel and tibia. Bone, 25(1), 49-54

Cenci, S., Weitzmann, M. N., Roggia, C., Namba, N., Novack, D., Woodring, J., et al. (2000). Estrogen deficiency induces bone loss by enhancing T-cell production of TNF-a. Journal of Clinical Investigation, 106(10), 9.

Colado, J. C., Triplett, N. T., Tella, V., Saucedo, P., \& Abellán, J. (2009). Effects of aquatic resistance training on health and fitness in postmenopausal women. European Journal of Applied Physiology, $106,113-122$

Connelly, D. M., Rice, C. L., Roos, M. R., \& Vandervoort, A. A. (1999). Motor unit firing rates and contractile properties in tibialis anterior of young and old men. Journal of Applied Physiology, 87, 10.

Cottini, E., Rando, G., Cirino, A., Giunta, S., Giacone, G., Vintaloro, G., et al. (1996). Importance of training in prevention of the decline of physical performance in elderly sedentary persons and veteran athletes. Archieves of Gerontology and Geriatrics, 22, 6.

Cox, K. L., Burke, V., Beilin, L. J., Derbyshire, A. J., Grove, J. R., Blanksby, B. A., et al. (2008). Short and long-term adherence to swimming and walking programs in older women - The Sedentary WomenExerciseAdherence Trial(SWEAT2). Preventive Medicine, 46, 511-517.

Cox, K. L., Burke, V., Beilin, L. J., Grove, J. R., Blanksby, B. A., \& Puddey, B. (2006). Blood pressure ries with swimming versus walking in older women: the Sedentary Women Exercise Adherence Trial 2 (SWEAT 2). Journal of Hypertension, 24(2), 307-314.

Chirosa, L. J., Chirosa, I. J., Requena, B., Feriche, B., \& Padial, P. (2002). Efecto de diferentes métodos de entrenamiento de contraste para la mejora de la fuerza de impulsión en un saldo vertical. Revista Motricidad(8), 26. 
Daley, M. J., \& Spinks, W. L. (2000). Exercise, mobility and aging. Sports Medicine, 1, 1-12.

Deschenes, M. R., \& Kraemer,W.J.(2002). Performance and physiologic adaptations to resistance training. American Journal of Physical Medicine \& Rehabilitation, 81, S3-S16.

Ebben, W.P.(2002). Complex training: a brief review. Journal of Sports Science and Medicine, 1, 42-46.

Fiatarone, M. (2002). Benefits of exercise and dietary measures to optimize shifts in body composition with age. The American Journal of Clinical Nutrition., 11, 642-652.

Gehri, D. J., Ricard, M. D., Kleiner, D. M., \& Kirkendall, D. T. (1998) A comparison of plyometric training techniques for improving vertical jump ability and energy production. Journal of Strength and Conditioning Research, 12(2), 85-89.

Greeves, J. P., Cable, N. T., Reilly, T., \& Kingsland, C. (1999). Changes in muscle strength in women following the menopause: a longitudinal assessment of the efficacy of hormone replacement therapy. Clinical Science, 97, 79-84

Hans, D., Hartl, F., \& Krieg, M. A. (2003). Device-specific weightered T-score for two quantitative ultrasounds: operational propositions for the management of osteoporosis for 65 years and older women in Switzerland. Osteoporosis International, 14, 251-258.

Humphries, B., Triplett-McBride, T., Newton, R. U., Marshall, S., Bronks, R., McBride, J., et al. (1999). The relationship between dynamic, isokinetic and isometric strength and bone mineral density in a population of 45 to 65 year old women. Journal of Science and Medicine in Sport, 2(4), 11.

Kamel, H. K., Maas, D., \& Duthie, E. H. (2002). Role of hormones in the pathogenesis and management of sarcopenia. Drugs Aging, 19(11), 13

Keene, G. S., Parker, M. J., \& Pryor, G. A. (1993). Mortality and morbidity after hip fractures. British Medical Journal, 307, 12481250.

Kell, R. T., Bell, G., \& Quinney, A. (2001). Musculoeskeletal fitness, health outcomes and quality of life. Sports Medicine, 31(12), 11.

Kent-Braun, J. A., \& Ng, A. V. (1999). Specific strength and voluntary muscle activation in young and elderty women and men. Journal of Applied Physiology, 87, 22-29.

Klass, M., Baudry, S., \& Duchateau, J. (2008). Age-related decline in rate of torque development is accompanied by lower maximal motor unit discharge frequency during fast contractions. Journal of Applied Physiology, 104, 739-746.

Laskin, D., Costlow, J., López, M. C., Taub, M., \& Kronenberg, F. (1994). Menopausia. La entrada a nuestra tercera edad. In P. Brown \& D. Laskin (Eds.), Envejecer juntas. Las mujeres y el paso del tiempo: datos para afrontarlo con optimismo, conocimiento de causa y decisión. (pp. 514). Barcelona: Ediciones Paidós Ibérica, S.A.

Markovic, G. (2007). Does plyometric training improve vertical jump height? Ameta-analytical review. British Journal of Sports Medicine, 41(6), 349-355.

Martel, G. F., Harmer, M. L., Logan, J. M., \& Parker, C. B. (2005). Aquatic plyometric training increases vertical jump in female volleyball players. Medicine and Science in Sports and Exercise, 37(10), 1814-1819.

Meunier, P. J., Delmas, P. D., Eastell, R., McClung, M. R., Papapoulos, S., Rizzoli, R., et al. (1999). Diagnosis and management of osteoporosis in postmenopausal women: clinical guidelines. Clinical Therapeutics, 21(6), 20.

Muntané, M. D. (1994). La Menopausia. Cómo afecta a las mujeres y cómo resolverla. Barcelona: Icaria.

Pollock, M. L., Gaesser, G A., Butcher, J. D., Després, J.-P., Dishman, R. K., Franklin, B. A., et al. (1998). The recommended quantity and quality of exercise for developing and maintaining cardiorespiratory and muscular fitness and flexibility in healthy adults. Medicine and Science in Sports and Exercise, 30(6), 17.
Potteiger, J. A., Lockwood, R. H., Haub, M. D., Dolezal, B. A., Almuzaini, K. S., Schroeder, J. M., et al. (1999). Muscle power and fiber characteristics following 8 weeks of plyometric training. Journal of Strength and Conditioning Research, 13(3), 275-279.

Riggs, B. L., Khosla, S., \& Melton, L. J. (1998). A unitary model for involutional osteoporosis: estrogen deficiency causes both type I and type II osteoporosis in postmenopausal women and contributes to bone loss in aging men. Journal of Bone and Mineral Research, 13(5), 11.

Runge, M., \& Schacht, E. (2005). Multifactorial pathogenesis of falls as a basis for multifactorial interventions. Journal of Musculoskeletal and Neuronal Interactions, 5(2), 8.

Sipilä, S., Taaffe, D. R., Cheng, S., Puolakka, J., Toivanen, J., \& Suominen, H. (2001). Effects of hormone replacement therapy ans high-impact physical exercise on skeletal muscle in post-menopausal women: a randomized placebo-controlled study. Clinical Science, 101, 10.

Stewart, A., Kumar, V., \& Reid, D. M. (2006). Long-term fracture prediction by DXA and QUS: a 10-year prospective study. Journal of Bone and Mineral Research, 21(3), 413-418.

Taaffe, D. R., Sipilä, S., Cheng, S., Puolakka, J., Toivanen, J., \& Suominen, H. (2005). The effect of hormone replacemen therapy and/or exercise on skeletal muscle attenuation in postmenopausal women: ayearlong intervention. Clinical Physiology and Functional Imaging., 25, 297304.

Takeshima, N., Rogers, M. E., Watanabe, E., Brechue, W.F., Okada, A., Yamada, T., et al. (2002). Water-based exercise improves healthrelated aspects of fitness in older women. Medicine and Science in Sports and Exercise, 34(3), 8.

Tsourlou, T., Benik, A., Zafeiridis, A., \& Kellis, S. (2006). The effects of a twenty-four week aquatic training program on muscular strength performance in healthy elderly women. Journal of Strength and Conditioning Research, 20(4), 8.

Van Langendonck, L., Claessens, A. L., Lysens, R., Koninckx, P. R., \& Beunen, G. (2004). Association between bone, body composition and strength in premenarcheal girls and postmenopausal women. Annals of Hyman Biology, 31(2), 26.

Van Pelt, R. E., Davy, K. P., Stevenson, E. T., Wilson, T. M., Jones, P. P., Desouza, C. A., et al. (1998). Smaller differences in total and regional adiposity with age in women who regularly perform endurance exercise. American Journal of Physiology, 275, E626E634.

Vanezis, A., \& Lees, A. (2005). A biomechanical analysis of good and poor performers of the vertical jump. Ergonomics, 48(11-14), 1594-1603.

Vilijanen, T., Viitasalo, J. T., \& Kujala, U. M. (1991). Strength characteristics of a healthy urban adult population. European Journal of Applied Physiology \& Occupational Physiology, 63(1), 5.

Volaklis, K. A., Spassis, A. T., Tokmakidis, S. P., \& Greece, K. (2007). Land versus water exercise in patients with coronary artery disease: effects on body composition, blood lipids and physical fitness. American Heart Journal, 154, 6.

WHO, \& TUSNP. (2002). Keep fit for life. Meeting the nutritional needs of older persons. [Electronic Version]. World Health Organization \& Tufts University School of Nutrition and Policy. http://whqlibdoc.who.int/publications/9241562102.pdf. Retrieved 21-06-07,

Wilmore, J. H., \& Costill, D. L. (2004). Fisiología del esfuerzo y del deporte ( $5^{\mathrm{a}}$ ed.). Barcelona: Editorial Paidotribo.

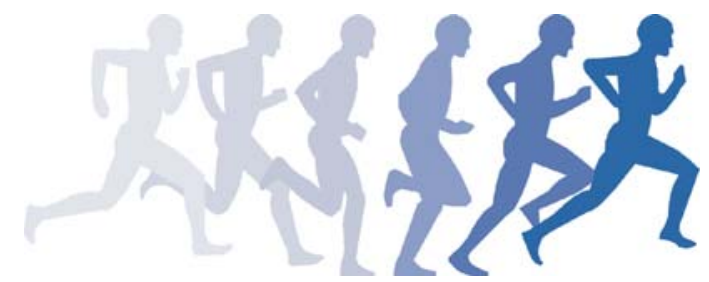

